\title{
A Technique for the Optical Characterization of Imaging Air-Cherenkov Telescopes
}

\author{
F. Arqueros, G. Ros, G. R. Elorza and D. García-Pinto \\ Departamento de Física Atómica, Molecular y Nuclear, Facultad de Ciencias Físicas, Universidad Complutense, \\ E-28040 Madrid, Spain \\ Presenter: F. Arqueros (arqueros@gae.ucm.es), spa-arqueros-F-abs1-og27-oral
}

A procedure for the optical characterization of imaging air-Cherenkov telescopes IACTs is discussed. A CCD camera with a lens, located near the telescope focus records images of the mirror while the pointing direction of the telescope moves around a star. For each image, the slope of those mirror points which are recorded bright, due to the light star, can be accurately measured by the simple application of the reflection law. This procedure allows the reconstruction of the slope map of the mirror. A simple simulation shows that the slope map could be reconstructed with an uncertainty better than $0.1 \mathrm{mrad}$.

\section{Introduction}

Imaging air-Cherenkov telescopes IACT have proof to be the most sensitive technique for the detection of very high energy $\gamma$-ray sources [1]. These telescopes use a large mirror (usually tessellated) for focusing the air-Cherenkov light generated by the the shower onto a PMT camera located at the focal plane. The angular size of the image for a point source is a very important parameter since a broad light spot leads to a loss of angular resolution, reducing consequently the capabilities for hadronic background rejection. The features of the light spot are mainly determined by aberrations, optical quality of individual facets and facet alignment. A useful method for checking both facet alignment and mirror quality relies in the analysis of the light spot of a star at the focal point. Unfortunately the size and shape of the light spot do not provide accurate information on the origin of a possible problem (e.g. misaligned facets, poor quality of the mirror or deformations of the telescope structure).

In this paper a procedure is presented which allows the direct measure of the unit vector normal to the mirror surface (slope) at any point of the telescope using a very simple set-up. More details on this technique can be found in [2]. This so-called SCCAN technique, first developed for solar concentrators, has been tested with the heliostats of a solar plant [3].

\section{The SCCAN technique}

The image of an ideal paraboloidal mirror for a point source (object at infinity) on the optical axis is a geometrical point at the focus. Therefore an observer located at the focus would see the whole mirror surface "bright" (i.e. light comes from all mirror points). Because a realistic mirror is far from ideal (optical aberrations, imperfections of the mirror surface), the image of a point source is not a geometrical point but a light spot LS.

Assuming that the diameter of the observer window $d$ (i.e. the entrance pupil of the observer) is much smaller than the LS, only certain regions of the mirror surface would be seen "bright" by an observer located at the LS, in fact only those points of the mirror surface for which the observer location fulfills precisely the reflection law. For an observer moving inside the LS every mirror point will be seen "bright" from some position.

Assuming that $d$ is much smaller than the focal length of the mirror $f$, the position of the "bright" spots allows 


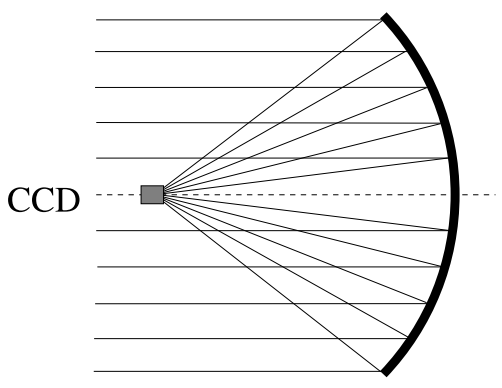

a)

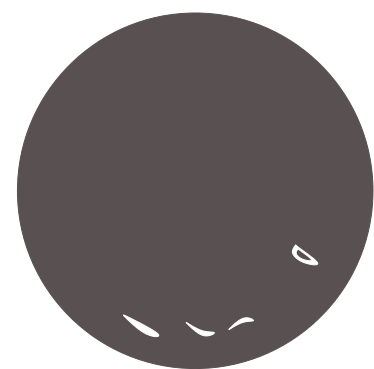

c)

b)

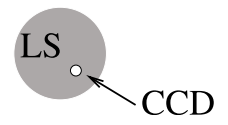

Figure 1. Schematic diagram of the SCCAN technique applied to an IACT. (a) The light from a point source is focused by the mirror in the light spot where a CCD with the appropriate lens is located. (b) The entrance of the CCD is located inside the light spot LS. (c) A typical picture of the mirror containing bright spots due to the reflected light. For each picture the slope of the mirror can be reconstructed at the bright spots. Scanning the LS around the CCD allows to measure the whole slope map of the mirror.

a precise measurement of the unit vector normal to the surface by a simple application of the law of reflection. Assuming a perfect knowledge of the location of the "bright" spots on the mirror, the angular position of the point source and the location of the observer, the theoretical limit for the uncertainty of the slope measurement is of the order of $d / f$ radians. In fact the slope can also be reconstructed from outside the focal plane as far as the observer is crossed by a sufficient fraction of the ray paths.

The most natural light source for these measurements is a star although an artificial source at a large distance can also be used. For a practical and fast data taking, a CCD camera provided with the appropriate lens to record images of the mirror can be used (see figure 1). In order to characterize the whole mirror surface, the camera has to take images at many positions by scanning the whole LS. For IACTs with an accurate tracking system, a very simple procedure is to move the pointing direction of the telescope around the direction of the star. This allows the relative movement of the CCD camera with respect to the LS. It is also possible to keep a fixed direction of the telescope and let the LS to cross the CCD. Successive scans can be programmed with shifts in the telescope direction perpendicular to the star movement. Many stars can be used for these measurements and thus the mirror can be characterized at any direction of interest. This is very useful for checking possible deformations of the mirror surface due to mechanical strains at certain aiming directions.

Note that in the usual technique for the mirror characterization, a camera located near the mirror center records the LS of a point source focused onto a screen located at the focal plane. Instead, in our technique, the CCD is located near the focal point and records many images of the mirror with those bright points while the source is moving around the pointing direction of the telescope. While the analysis of the light density distribution (dependent on aberrations) only provides a rough estimate of the optical quality of the mirror, our technique allows the direct measure of the slope map. It is also important noting that the SCCAN technique is only based in the reflection law and no assumption on the optical properties of the mirror (for instance, aberrations) has to be made. A mirror point which is observed bright for a given position of the source has a slope easily calculated 
from the reflection law, no matter if that slope value is due to mirror imperfections, facet misalignment or just the expected one from a perfect mirror taking into account aberrations.

\section{Application to IACTs}

The capabilities of this technique for IACTs have been studied by means of a simulation. A single-piece parabolic mirror $17 \mathrm{~m}$ diameter, $17 \mathrm{~m}$ focal length with a light spot diameter (for on-axis point source) of about $3.0 \mathrm{~cm}$ has been assumed. An imperfect mirror surface has been simulated by adding a random perturbation to an ideal paraboloid with the optical axis along the $\mathrm{z}$ axis (more details in [2]). Rays hitting the mirror surface have been randomly generated in the direction of the optical axis uniformly distributed on the x-y plane. For each incident ray, the unit vector normal to the realistic surface in the hitting point has been calculated and the corresponding reflected ray has been computed. This information has allowed us to obtain the LS features, the expected images recorded by a CCD camera located at any position near the focal point and the capabilities of this technique for the reconstruction of the slope map.

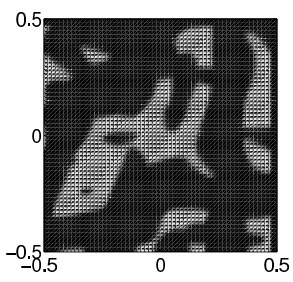

a)

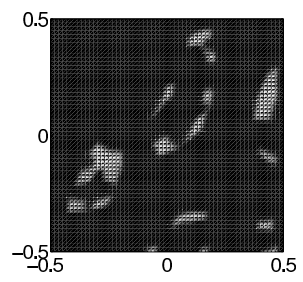

b)

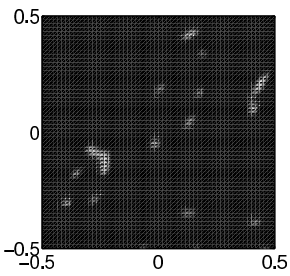

c)

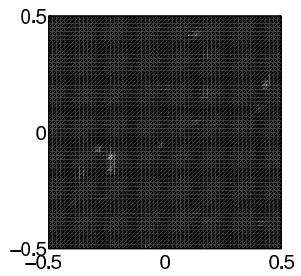

d)

Figure 2. Simulated images of the central region of the mirror $\left(1 \times 1 \mathrm{~m}^{2}\right)$ for $d$ values of $2 \mathrm{~cm} \mathrm{(a),} 1 \mathrm{~cm}(\mathrm{~b}), 0.5 \mathrm{~cm}(\mathrm{c})$ and $0.2 \mathrm{~cm}(\mathrm{~d})$.

As an example, figure 2 shows simulated images of the mirror recorded by a CCD+lens located near the center of the LS for several values of the entrance pupil diameters. As expected the size of the bright spots diminishes at lower $d$ values with improved accuracy in the slope reconstruction. For this example a mirror model with imperfections size of about $20 \mathrm{~cm}$ (equation (2) in [2]) has been used. The unit vector normal to any point of the mirror surface can be easily calculated from the measured incident and reflected unit vectors (simple application of the reflection law). Other important parameter is the accuracy in the position of the bright spot in the local coordinate system of the mirror. In practice the uncertainty in this position may be due to many contributions. In our simulation we will assume that the main contribution is given by the finite size of the pixel field of view. The normal vector map has been calculated after a uniform random shift of the direction of the reflected ray taking into account the pixel size. Values of the rms deviations of the reconstructed unit vector from the real one (see equation (5) in [2]) of 0.2 and $0.1 \mathrm{mrad}$ have been obtained for resolutions of $1000 \times 1000$ and $2000 \times 2000$ respectively. These results include the effect of a $2 \mathrm{~mm}$ diameter entrance pupil.

Figure 3 shows the map of slope errors obtained from a large series of images recorded by a $2000 \times 2000$ pixels CCD. The surface has been divided in cells of $10 \times 10 \mathrm{~cm}^{2}$. For each cell, the average error has been calculated using the information of all the rays hitting the cell. The uncertainty in the average values of the slope is of about $0.01 \mathrm{mrad}$ for an statistics of $100 \mathrm{hits} / \mathrm{cm}^{2}$.

As already mentioned, the CCD can be located ahead the PMT camera. The reconstruction of the slope is not affected as far as the reflected vector is properly calculated taken into account the real position of the CCD. 


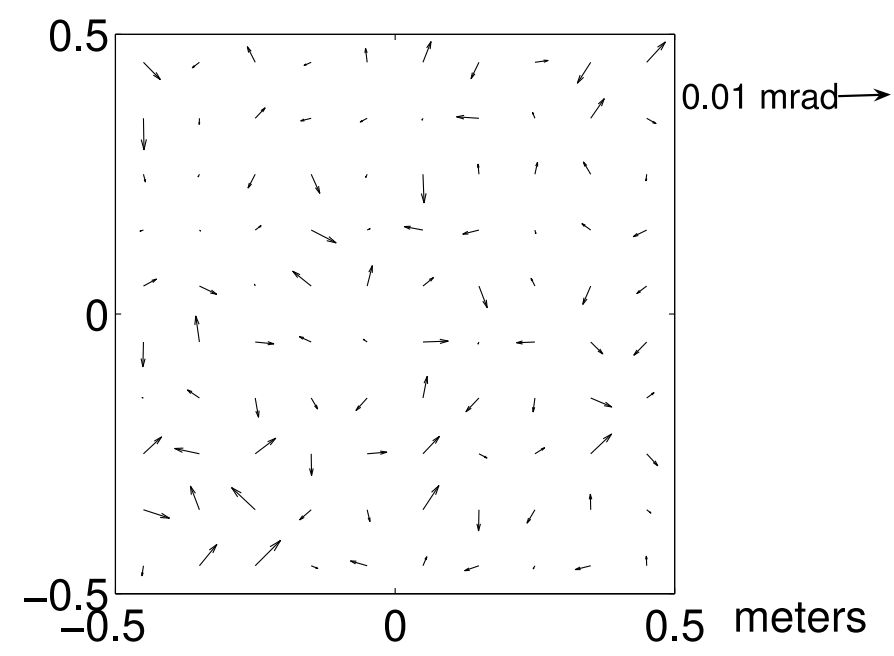

Figure 3. Map of reconstruction errors for the central region of the mirror. The arrows represent slope errors averaged in cell of $10 \times 10 \mathrm{~cm}^{2}$.

\section{Conclusions}

The applicability of the SCCAN technique for the optical characterization of IACTs has been discussed. The main advantage of SCCAN is the capability for the accurate measure of the slope map of the mirror surface in operative conditions and for any aiming direction of the telescope. The set-up is simple and non-expensive. An uncertainty better than $0.1 \mathrm{mrad}$ can be achieved in a few hours of data taking. This technique can be particularly useful for the detection of a facet misalignment or deformations of the mirror structure.

\section{Acknowledgements}

This work has been funded by the Spanish Ministerio de Ciencia y Tecnologia (ref.: FPA03-08733-C02-01). G. Ros acknowledges an undergraduate grant (Beca colaboración) from the Spanish Ministry of Education. Interesting comments of E. Lorenz and R. Mirzoyan are acknowledged.

\section{References}

[1] J.A. Hinton et al., New Astron.Rev. 48 (2004) 331; C. Baixeras et al., Nucl.Instrum.Meth.A 518 (2004) 188; Weekes T. C., et al., Astropart. Phys. 17 (2002) 221; Kawachi et al., Astropart. Phys. 14 (2001) 261.

[2] F. Arqueros et al., Astropart. Phys. (in press).

[3] F. Arqueros et al., Solar Energy 75 (2003) 135. 